\title{
An Experimental Setup for Performance Analysis of an Online Adaptive Cooperative Spectrum Sensing Scheme for Both In-Phase and Quadrature Branches
}

\author{
Serhan Yarkan*, Khalid. A. Qaraqe*, B. Uğur Töreyin*, A. Enis Çetin ${ }^{\S}$ \\ *Electrical \& Computer Engineering, Texas A \& M University

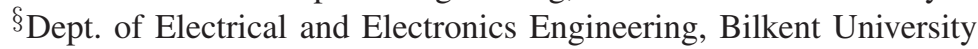 \\ E-mails: syarkan@ece.tamu.edu, khalid.qaraqe@qatar.tamu.edu, \\ behcet.toreyin@qatar.tamu.edu, cetin@bilkent.edu.tr
}

\begin{abstract}
Spectrum sensing is one of the most essential characteristics of cognitive radios (CRs). Robustness and adaptation to varying wireless propagation scenarios without compromising the sensing accuracy are desirable features of any spectrum sensing method to be deployed in CR systems. In this study, an online adaptive cooperation technique for spectrum sensing is proposed in order to maintain the level of reliability and performance. Cooperation is achieved by sensors which employ energy detection. These sensors send their output to a center where data fusion operation is carried out in an online and adaptive manner. Adaptation is realized by the use of orthogonal projections onto convex sets (POCS). In conjunction with the proposed method, an end-to-end methodology for a flexible experimental setup is also proposed in this study. This setup is arranged to emulate the proposed adaptive cooperation scheme for spectrum sensing and validate its practical use in cognitive radio systems. Comparative performance results for both inphase and quadrature branches are presented.
\end{abstract}

Index Terms-projection onto convex sets, cognitive radio systems, adaptive data fusion, online learning, experimental setup, wireless propagation

\section{INTRODUCTION}

Cognitive radio (CR) systems, which are aware of their surroundings with self-adaptation capability to dynamic environmental and channel conditions, have emerged as a novel paradigm in wireless communications [1]. One of the most distinguished features of these systems is spectrum sensing for dynamic spectrum access. It has been reported repeatedly in the literature that cooperation among spectrum sensors substantially increases the overall performance and sensing reliability of a $\mathrm{CR}$ system [2, 3]. Furthermore, cooperative spectrum sensing has been shown to mitigate the negative effects that stem from practical problems like multipath fading/shadowing and noise power fluctuations by exploiting the diversities among multiple CRs (spectrum sensors) [3].

The literature on cooperative spectrum sensing has been exponentially growing for the last couple of years [3]. However, existing methods are developed based on a static communication scenario assumption with fixed channel and radio frequency (RF) propagation environment characteristics. In addition to that, reports on practical implementations of such models are limited. In this study, we not only propose an adaptive data fusion (ADF) scheme for cooperative spectrum sensing, which exploits inherent dynamics of the sensing problem by updating the weight of the contribution from each spectrum sensor in an online manner, but also we develop an end-to-end methodology for a flexible experimental setup.

The proposed cooperative spectrum sensing scheme comprises of a fixed number of sensors. Each sensor carries out conventional energy detection operation. Outputs of energy detectors are sent to a fusion center for their corresponding weights to be updated online based on the ADF scheme through the use of orthogonal projections onto convex sets (POCS). Similar online learning approaches based on POCS with their adaptation power for a system in which data arrives sequentially have been developed recently for several signal and image processing applications $[4,5]$.

The experimental setup is specifically deployed to emulate the proposed adaptive cooperation scheme for spectrum sensing and validate its practical use in cognitive radio systems. On the other hand, as shown in the sequel, proposed methodology and experimental setup may be implemented to investigate other system models developed for different practical scenarios with slight modifications. Another important contribution of this paper is that, both in-phase and quadrature branches of the communication system between the sensors and the fusion center are analyzed experimentally.

The organization of the paper is as follows. The statement of the problem and the system model are presented in Section II. In Section III, the proposed online adaptation and data fusion method is described. Details of the experimental setup, data collection, and processing are given in Section IV. Numerical results and discussions with an emphasis on inphase and quadrature branches are presented in Section V. Finally, conclusions are drawn in the last section.

\section{System Model and Statement of the Problem}

Let a fixed, immobile spectrum sensor network be composed of $M$-many sensors. Each sensor, Sensor ${ }_{i}$, where $i=$ $1, \ldots, M$, carries out a sequence of analysis steps described in the sequel upon receiving a signal $r_{i}(\cdot)$ as shown in Figure 1. The baseband equivalent of the received signal at the $i$-th 


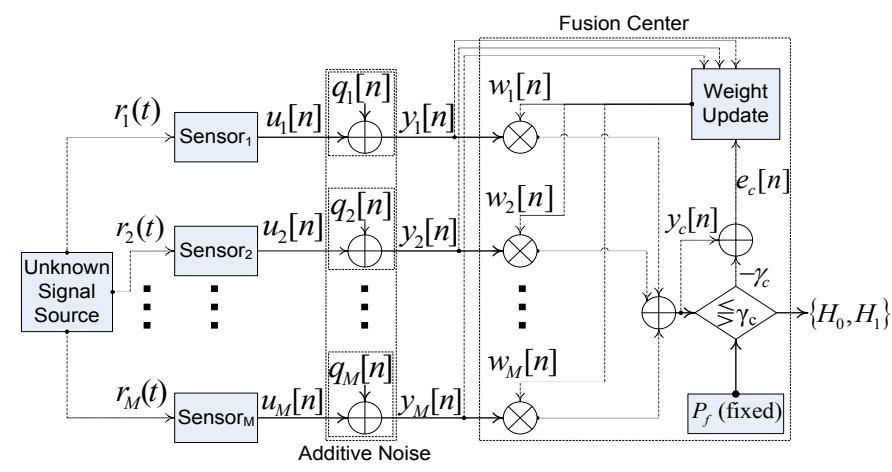

Fig. 1. System model for the online adaptive cooperative spectrum sensing.

sensor, $r_{i}(\cdot)$, can be represented as:

$$
r_{i}(t)=\left\{\begin{array}{rr}
v_{i}(t), & H_{0}, \\
x(t)+v_{i}(t), & H_{1},
\end{array}\right.
$$

where $v_{i}(t)$ is complex additive white Gaussian noise (AWGN) with $\mathcal{C N}\left(0, \sigma_{i}^{2}\right)$ (both real and imaginary parts having the same variances); $x(t)$ is the complex baseband equivalent of the unknown signal transmitted by a single source; $H_{0}$ corresponds to the hypothesis for the absence of the unknown signal, whereas $H_{1}$ refers to the hypothesis corresponding to the presence of it at time $t$. Then, the problem can be stated as identifying the absence/presence of the unknown signal, $x(t)$, by taking into account dynamic changes in the channel and the propagation environment that possibly include practical design issues and physical phenomena as shadowing, fading, mobility, and so on.

Each spectrum sensor, Sensor $i$, yields a summary statistic $u_{i}$, where $i=1, \ldots, M$. At this point, it is worth mentioning that the output of each sensor can be considered to be of discrete form rather than a continuous form such as $u_{i}[n]$ for the sake of notational convenience in the subsequent steps. Summary statistic at $n$-th discrete time instant, namely $u_{i}[n]$, is calculated by each secondary user over a detection interval of $N$ samples as follows:

$$
u_{i}[n]=\sum_{k=n-N+1}^{n}\left|r_{i}[k]\right|^{2} \quad i=1,2, \ldots, M
$$

These data are transmitted over a very narrow-band channel, say "Broadcast Fusion Channel," to an immobile "fusion center." In this regard, since sensors and fusion center are all assumed to be fixed, the set of signals reaches at the fusion center at $n$-th discrete time instant can be modeled as a Gaussian channel with zero-mean noise $q_{i}$ and with variance $\delta=\left[\delta_{1}^{2}, \delta_{2}^{2}, \ldots, \delta_{M}^{2}\right]^{T}$ :

$$
\mathbf{y}[n]=\mathbf{u}[n]+\mathbf{q}[n]
$$

with $\mathbf{y}[n]=\left[y_{1}[n], \ldots, y_{M}[n]\right]^{T}, \quad \mathbf{u}[n] \quad=$ $\left[u_{1}[n], \ldots, u_{M}[n]\right]^{T}$ and $\mathbf{q}[n]=\left[q_{1}[n], \ldots, q_{M}[n]\right]^{T}$. At the fusion center, where the adaptive data fusion is realized online, a global test statistic $y_{c}[n]$ is computed from $y_{i}[n]$. Carrying out analysis steps similar to the one presented in [2], the following linear rule at the fusion center with a test threshold $\gamma_{c}[n]$ is obtained:

$$
y_{c}[n] \gtreqless \mathcal{H}_{1} \gamma_{c}[n]
$$

where

$$
y_{c}[n]=\sum_{i=1}^{M} w_{i}[n] y_{i}[n]=\mathbf{y}[n] \mathbf{w}[n]
$$

and

$$
\mathbf{w}[n]=\left[w_{1}[n], \ldots, w_{M}[n]\right]^{T}, \quad w_{i}[n] \geq 0
$$

The performance metric used for the system model is the following $\left\{P_{f}, P_{d}\right\}$ pair:

$$
P_{f}=Q\left[\frac{\gamma_{c}-N \sigma^{\mathbf{T}} \mathbf{w}}{\sqrt{\mathbf{w}^{\mathbf{T}} \mathbf{\Sigma}_{\mathcal{H}_{0}} \mathbf{w}}}\right]
$$

and

$$
P_{d}=Q\left[\frac{\gamma_{c}-\left(N \sigma+\mathbf{E}_{\mathbf{s}} \mathbf{g}\right)^{\mathbf{T}} \mathbf{w}}{\sqrt{\mathbf{w}^{\mathbf{T}} \mathbf{\Sigma}_{\mathcal{H}_{1}} \mathbf{w}}}\right]
$$

where $P_{f}$ denotes the probability of false alarm and $P_{d}$ denotes the probability of detection, and $Q($.$) is the complementary$ cumulative distribution function, which calculates the tail probability of a zero mean unit variance Gaussian random variable, $\sigma=\left[\sigma_{1}, \ldots, \sigma_{\mathbf{M}}\right], \boldsymbol{\Sigma}_{\mathcal{H}_{0}}$ and $\boldsymbol{\Sigma}_{\mathcal{H}_{1}}$ are system parameter matrix defined in [2]. For the sake of brevity, the sample number index, $n$, is removed in (7) and (8).

Here note that different weight selection rules can be employed for different purposes, such as the one described in $[6,7]$. In this study, although the broadcast fusion channel is assumed to be of AWGN form, there is no restriction imposed on the channel (and on the type of propagation environment such as urban or suburban) between the sensors and the unknown signal source. Therefore, the system model can easily be extended to the one in which unknown signal $x(t)$ can be assumed to undergo shadowing, multipath fading, and Doppler spread (i.e., a mobile unknown signal source) as well.

\section{OnLine AdAPtive CoOperation Scheme}

Let $\mathbf{w}[n]=\left[w_{1}[n], \ldots, w_{M}[n]\right]^{T}$ be the weight vector at each spectrum sensor at time step $n$. Then we define

$$
y_{c}[n]=\mathbf{y}[n] \mathbf{w}[n]=\sum_{i} w_{i}[n] y_{i}[n]
$$

as an estimate of the test threshold, $\gamma_{c}[n]$, at time step $n$, and the error, $e_{c}[n]=\gamma_{c}[n]-y_{c}[n]$. For a fixed value of probability of false alarm, $P_{f}$, one can obtain the corresponding threshold value, $\gamma_{c}[n]$, from (7) as:

$$
\gamma_{c}=N \sigma^{\mathbf{T}} \mathbf{w}+\mathbf{Q}^{-\mathbf{1}}\left(\mathbf{P}_{\mathbf{f}}\right) \sqrt{\mathbf{w}^{\mathbf{T}} \boldsymbol{\Sigma}_{\mathcal{H}_{0}} \mathbf{w}}
$$

At each time step $n, \gamma_{c}[n]$ is evaluated using (10), for a fixed $P_{f}$ value and the weight vector, $\mathbf{w}[n]$. Along with the weight update equation presented in the next subsection, (10) provides weights adapt themselves in such a way that statistics of $P_{f}$ is not affected from dynamic changes and drifts in the channel and/or the propagation environment. This is achieved via the controlled feedback mechanism based on the error term, $e_{c}[n]$. 
Ideally, weighted sum of the received summary statistics of spectrum sensors should be equal to the test threshold $\gamma_{c}[n]$ :

$$
\gamma_{c}[n]=\mathbf{y}^{T}[n] \mathbf{w}
$$

which represents a hyperplane in the $M$-dimensional space, $\mathbf{w} \in \mathbb{R}^{M}$. Hyperplanes are convex in $\mathbb{R}^{M}$. At time instant $n, \mathbf{y}^{T}[n] \mathbf{w}[n]$ may not be equal to $\gamma_{c}[n]$. The next set of weights are determined by projecting the current weight vector $\mathbf{w}[n]$ onto the hyperplane represented by (11). The orthogonal projection $\mathbf{w}[n+1]$ of the vector of weights $\mathbf{w}[n] \in \mathbb{R}^{M}$ onto the hyperplane $\gamma_{c}[n]=\mathbf{y}^{T}[n] \mathbf{w}$ is the closest vector on the hyperplane to the vector $\mathbf{w}[n]$.

Let us formulate the problem as a minimization problem:

$$
\min _{\mathbf{w}^{*}}\left|\mathbf{w}^{*}-\mathbf{w}[n]\right|, \quad \text { subject to: } \mathbf{y}^{T}[n] \mathbf{w}^{*}=\gamma_{c}[n]
$$

Solution can be obtained by using Lagrange multipliers:

$$
\mathcal{L}=\sum_{i}\left(w_{i}[n]-w_{i}^{*}\right)^{2}+\lambda\left(\mathbf{y}^{T}[n] \mathbf{w}^{*}-\gamma_{c}[n]\right)
$$

Solving this minimization problem, weight update equation can be obtained as [8]:

$$
\mathbf{w}[n+1]=\mathbf{w}[n]+\mu \frac{e_{c}[n]}{\|\mathbf{y}[n]\|^{2}} \mathbf{y}[n]
$$

where $\mu$ is a relaxation parameter for which $0<\mu<2$ should be satisfied to guarantee the convergence according to the POCS theory $[9,10]$.

Here, note that, the method does not need to wait for weights to converge to give a decision. At each time step $n$, the updated weight vector simply tracks test threshold, $\gamma_{c}[n]$, by assigning proper weights to individual spectrum sensors, in order to maintain the same $P_{f}$ value under dynamically changing channel and propagation environment characteristics.

\section{Experimental Setup, Data Collection and PROCESSING}

The experimental setup was deployed at the Wireless Research Laboratory located in the Department of Electrical and Computer Engineering, Texas A\&M University at Qatar, Doha. A mainframe in the network to which all other devices used in the experiment connected is used for data collection, storage, and processing purposes. Further details of the experimental setup, devices, and equipments used will be given in this section along with the methodology employed in data collection and processing.

\section{A. Experimental Setup, Devices, and Equipments Used}

In accordance with the system model in Figure 1, the whole experiment consists of two parts: (P.1) the part where the unknown signal source is received by the sensors and processed and (P.2) the part where the output of sensors are transmitted to and fused by the ADF center, and the final decision is made. In the system model, (P.1) represents a conventional wireless propagation environment, whereas (P.2) assumes an AWGN channel. In order to emulate AWGN channel assumption, a direct cable (wired) connection is established between transmitter-receiver pair.

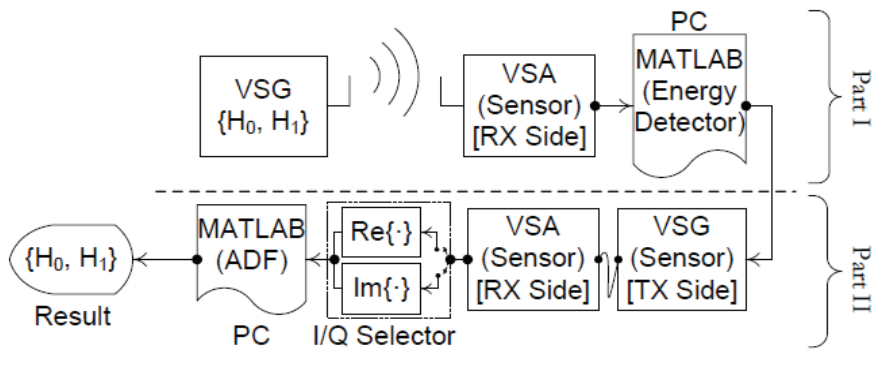

Fig. 2. Block diagram of the experimental setup emulating the system model given in Figure 1. The dashed horizontal line separates the implementation parts of the experiment.

All of the transmitters (i.e., unknown source and sensors) in the experiments are emulated by Agilent vector signal generator (VSG) E4438C. The VSG E4438C can be programmed and controlled through the use of a computer and of a software running on such as MATLAB. Therefore, a single VSG is used in time division multiple access (TDMA) fashion for emulating all of the transmitter units present in the setup. Similar to the VSG, Agilent PSA Series vector signal analyzer (VSA) E4440A is used in TDMA fashion for emulating all of the receiver units (i.e., sensors and ADF center) present in the setup. The block diagram of the whole experimental setup is sketched in Figure 2.

\section{B. Experimental Methodology and Data Collection}

In (P.1), each sensor is emulated in a TDMA fashion and received baseband signal (the output of VSA as inphase/quadrature (I/Q) samples) is stored on a computer to be fed into MATLAB. For the hypothesis $H_{0}$, the unknown signal source (i.e., VSG) is deactivated so that receiver side (denoted as "rx-side" in Figure 2) of each sensor that is emulated by VSA captures the ambient noise over the air. For the hypothesis $H_{1}$, the VSG is programmed to transmit a finite random data (which already exist in the device memory) according to North American Digital Cellular (NADC) standard with the given transmit parameters in a continuous manner. Data corresponding to both hypotheses are stored for a very large period of time to obtain sufficient statistics. Stored data are, later on, split into chunks and organized in such a way that each chunk corresponds to an independent realization.

In (P.2), the organized baseband I/Q values for each hypothesis are put into the VSG memory through the use of the specific MATLAB script. Upon setting the parameters of the VSA accordingly to receive the data sent over the cable, the transmission begins and VSA starts to capture the signal and stores it on a computer to be fed into MATLAB on which ADF algorithm runs to generate the output. Finally, the output of $\mathrm{ADF}$ is obtained and results are shown and interpreted.

\section{Data Formatting and Processing}

In contrast to (P.1), a normalization step is performed in (P.2). This stems from the fact that the transmission loss causes the captured baseband I/Q values to be very small for VSG to deal with. However, prior to this normalization operation, 
synchronization should be established between VSG and VSA. Here it must be noted that even though both VSG and VSA could be perfectly synchronized with a cable connecting both devices (i.e., via external reference), such an approach is not adopted in this work since an experimental setup that emulates a realistic scenario is targeted. In this sense, synchronization step cannot be excluded as in many real-world applications. Before transmitting the output of each sensor through the use of VSG, output of energy detectors are put into the memory of VSG in the following format: $\mathbf{p}_{L_{1}+L_{2}}=\left[\mathbf{0}_{L_{1}}^{T} \mathbf{d}_{L_{2}}^{T}\right]^{T}$, where $\mathbf{0}_{L_{1}}$ represents the zero-padding operation with a zero-vector of length $L_{1}, \mathbf{d}_{L_{2}}$ denotes the output vector of length $L_{2}$ including the energy detector output for any sensor, $\mathbf{p}_{L_{1}+L_{2}}$ represents the packet format to be sent over the cable through the use of VSG, and $(.)^{T}$ denotes the transpose operation. Unless stopped, VSG keeps sending the information back to back. In order to guarantee that VSA captures at least one complete $\mathbf{d}_{L_{2}}$, capture time of VSA is set to three times the total duration of a single packet $\mathbf{p}_{L_{1}+L_{2}}$. With the aid of the packet format $\mathbf{p}$, burst detection is performed to synchronize the packet in which the data is placed. Next, by exploiting $\mathbf{0}_{L_{1}}$, noise power estimation and normalization steps mentioned earlier in this subsection are performed. Due to the clock mismatch between the VSG and VSA, a frequency drift is expected in the received signal. Therefore, a clock recovery algorithm is implemented to remove this drift present in the I/Q samples that are provided by VSA. As will be shown subsequently, the cable connection (i.e., experimental broadcast fusion channel) would not imply an ideal AWGN behavior at the received signal due to the inherent low-pass filtering operation performed by VSG beside some other impairments such as coupling. Nevertheless, after the burst detection stage, normalization, and clock recovery steps, one can assume that the output is a reasonable approximation to the output of the theoretical broadcast fusion channel given in the system model. Finally, the real or imaginary part of output data are fed into ADF in order to make a decision in regards to $H_{0}$ and $H_{1}$ hypotheses as shown in Figure 2 with "I/Q selector". In the next section, the performance results for both real and imaginary parts of the signal fed into ADF will be given.

\section{RESUlts AND Discussion}

The general parameters given in Table I is used in all set of experiments. In realizing the hypothesis $H_{0}$, the VSG is kept inactive so that the VSA captures the ambient noise over the air. In order to make sure that what VSA captures over the air is solely the ambient noise, an empty RF band is selected prior to the experiment. On the other hand, in realizing the hypothesis $H_{1}$, a pseudo-random sequence in VSG memory is chosen to be continuously transmitted in NADC format to emulate the unknown signal source. For (P.1), VSG is located at a place where optical line-of-sight (LOS) is present between the VSG and VSA. For each sensor, measurements are repeated in a TDMA fashion 5000 times to obtain as many independent realizations as possible.
TABLE I

GENERAL SET OF PARAMETERS USED IN THE EXPERIMENTAL SETUP.

\begin{tabular}{|r|l|}
\hline Parameter & Value \\
\hline$\Delta t_{s}$ & $10^{-5}$ s \\
\hline No. of sensors $(M)$ & 20 \\
\hline No. of samples $(N)$ & 20 \\
\hline Unknown signal type & NADC \\
\hline Realization of $\left\{H_{0}, H_{1}\right\}$ & Bernoulli trial with $\operatorname{Pr}$ (success) $=0.5$ \\
\hline
\end{tabular}

Upon the data collection for both hypotheses, ADF is fed by the experimental data in such a way that an independent realization of a Bernouilli random variable with $\operatorname{Pr}$ (success) = $\operatorname{Pr}$ (failure) determines whether the input to ADF is selected from $H_{0}$ data or from $H_{1}$ data. Note that such a selection scheme forms one of the most challenging spectrum sensing behaviors for $\mathrm{ADF}$, since Bernoulli random variable with unit success-failure ratio implies maximum uncertainty about the absence/presence of the unknown signal. It must be noted here that most of the practical communication systems (depending also on the traffic type carried) transmit signals in bursts implying continuous presence in terms of spectrum sensing. Nevertheless, for the sake of investigating performance of the proposed method under challenging conditions, maximum uncertainty about the absence/presence of the unknown signal is adopted.

Since the hypothesis $H_{0}$ constitutes the base for the method proposed, noise characteristics of (P.1) should be investigated. This way, especially when practical conditions are of interest, one can obtain clues about what sort of impairments would be introduced into the data. Due to space limitations, only estimates of the autocorrelation coefficients of the captured data under $H_{0}$ hypothesis will be investigated. The results are given in Figure 3. It is clear that autocorrelation estimates (unbiased) of the captured data exhibit correlation within the first $0.4 \mathrm{~ms}$. Assuming that the ambient noise over the air is AWGN, theoretically speaking, Figure 3 is actually the impulse response of the device under test (DUT), which is in this case the VSA. Therefore, it can be concluded that the VSA acts as a low-pass filter which introduces correlation and distorts the statistical characteristics of the noise. Importance of the correlation introduced by VSA will be clearer subsequently.

The output of ADF when it is fed by the experimental data (after the organization step mentioned in Section IV-C) is given in Figure 4. Note that Figure 4 contains both cases where the "I/Q selector" in Figure 2 determines the type of input as $\Re(\cdot)$ or as $\Im(\cdot)$, which represents the real and imaginary part of the VSA output. It is obvious that when I/Q selector decides to feed the ADF with $\Im(\cdot)$, probability of miss-detection vanishes down to zero even for very small values of probability of false alarm. This is not surprising since the direct cable connection provides a frequency-flat channel as explained earlier. Furthermore, the output of energy detectors are realvalued samples. Hence, after synchronizing and applying clock recovery, under $H_{1}$ hypothesis, ideally, imaginary part of the output of VSA should include nothing but the corresponding part of ambient noise present in the RF front-end of the VSA 
and the artifacts of the recovery algorithms applied. Therefore, from Figure 4, one can conclude that ADF performs very well under practical conditions.

It is important to note that, the correlation introduced by the VSA to the experimental data fed to the ADF method as shown in Figure 3 yields smaller error valueswhich in turn results in weight vectors tracking the test threshold with higher accuracy.

\section{Vi. Conclusion and Future Directions}

An online adaptive cooperative spectrum sensing scheme based on the POCS theory is proposed in order to maintain the performance and the reliability of spectrum sensing. Performance of the proposed online adaptive cooperation scheme is investigated by a set of data collected from an experimental setup analyzing both in-phase and quadrature branches of the broadcast fusion channel in a selective manner. Even though the experimental setup described in the paper is designated specifically for this particular scheme using energy detectors, many other system models involving different types of channels and environments can also be validated with slight modifications in the proposed end-to-end experimental methodology.

Results show that the proposed method yields reliable spectrum sensing decisions for both in-phase and quadrature branches based on probability of false alarm and probability of detection metrics. Results also assure that this method can be utilized under challenging spectrum usage scenarios such as a cognitive radio system with a primary unknown signal source transmitting according to a Bernoulli trial process.

The proposed experimental methodology can be extended to include a full-mobile scenario in which all of the units in the system model including sensors and fusion center can be assumed to be mobile. Even though the contemporary experimental devices seem to be bulky to emulate a fullmobile scenario, there are different ways of manipulating the received signal to include several propagation effects such as Doppler and multipath. As future work, a full-mobile scenario will be studied.

\section{ACKNOWLEDGMENT}

This work is supported by Qatar National Research Fund (QNRF) grant through National Priority Research Program (NPRP) No. 08-101-2-025. QNRF is an initiative of Qatar Foundation. The authors also would like to thank Wael Halbawi for his assistance during experiments.

\section{REFERENCES}

[1] J. Mitola, "Cognitive radio architecture evolution," Proceedings of the IEEE, vol. 97, no. 4, pp. 626-641, April 2009.

[2] Z. Quan, S. Cui, and A. H. Sayed, "Optimal linear cooperation for spectrum sensing in cognitive radio networks," IEEE J. Select. Topics in Sig. Processing, vol. 2, no. 1, pp. 28-40, Feb. 2008.

[3] K. B. Letaief and W. Zhang, "Cooperative communications for cognitive radio networks," Proceedings of the IEEE, vol. 97, no. 5, pp. 878-893, May 2009.

[4] B. U. Toreyin, "Fire detection algorithms using multimodal signal and image analysis," Ph.D. dissertation, Bilkent University, Ankara, Turkey, Jan. 2009.

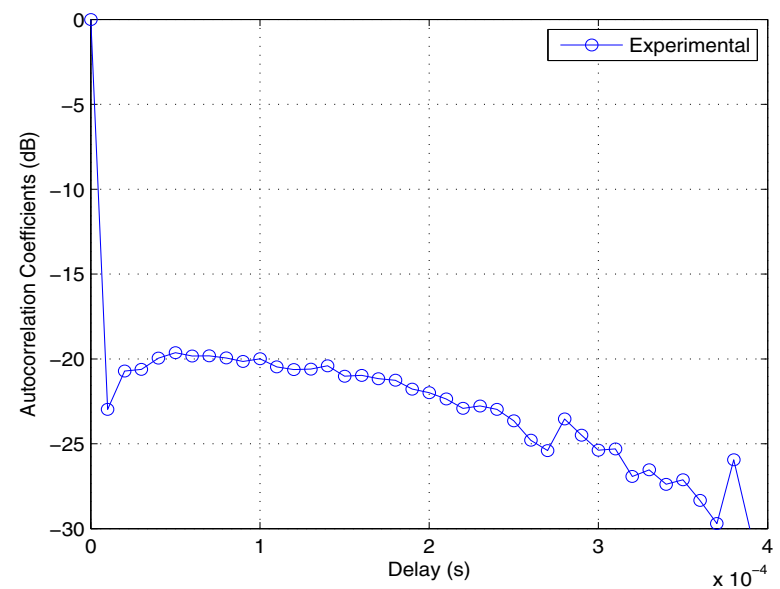

Fig. 3. Estimates of autocorrelation coefficients in logarithmic scale for experimental data. The impact of VSA filter manifests itself as a correlation during the first $0.4 \mathrm{~ms}$.

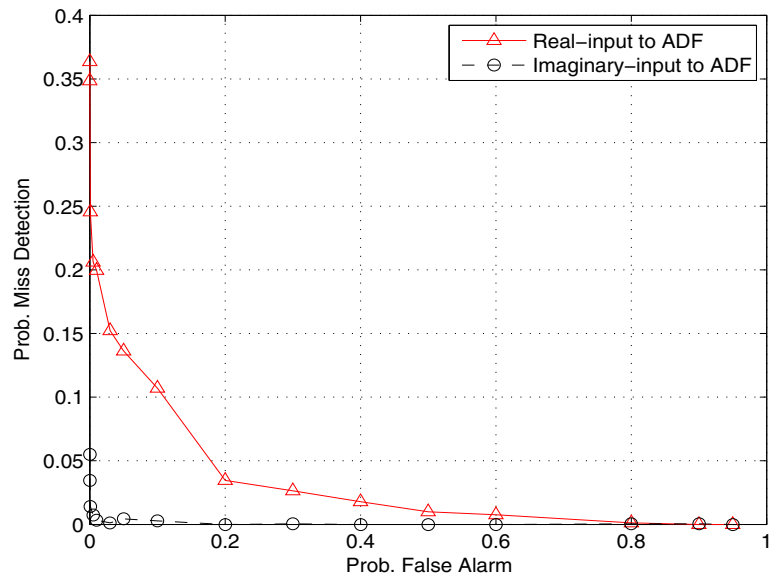

Fig. 4. The performance result of the proposed ADF mechanism with the experimental data collected for both $\Re(\cdot)$ and $\Im(\cdot)$ input in Figure 2.

[5] S. Theodoridis, K. Slavakis, and I. Yamada, "Adaptive learning in a world of projections," IEEE Signal Processing Magazine, vol. 28, no. 1, pp. 97-123, Jan. 2011.

[6] K. Zeng, P. Pawelczak, and D. Cabric, "Reputation-Based Cooperative Spectrum Sensing with Trusted Node Assistance," IEEE Communications Letters, vol. 14, no. 3, pp. 226-228, 2010.

[7] X. Zheng, J. Wang, Q. Wu, and J. Chen, "Cooperative spectrum sensing algorithm based on dempster-shafer theory," in 11th IEEE Singapore International Conference on Communication Systems (ICCS), 2008.

[8] S. Yarkan, B.U.Toreyin, K.A.Qaraqe, and A.E.Cetin, "An experimental validation of an online adaptive cooperation scheme for spectrum sensing," in International Conference on Telecommunications (ICT), May 2011, pp. 260-265.

[9] D. C. Youla and H. Webb, "Image restoration by the method of convex projections, part i-theory," IEEE Trans. on Med. Imaging, vol. MI-I-2, pp. 81-94, 1982.

[10] U. Niesen, D. Shah, and G. W. Wornell, "Adaptive alternating minimization algorithms," IEEE Trans. Inform. Theory, vol. 55, no. 3, pp. 1423-1429, March 2009. 